\title{
Conservation of a Fur Court Robe of the Qing Dynasty
}

\author{
Yunli Wang ${ }^{1}$, Chunlei Wang ${ }^{1}$ \\ ${ }^{1}$ The Palace Museum, Beijing, China \\ Correspondence: Yunli Wang, The Palace Museum, Beijing, China
}

Received: May13, $2015 \quad$ Accepted: May 27, $2015 \quad$ Online Published: July 20, 2015

doi:10.11114/set.v2i1.957 URL: http://dx.doi.org/10.11114/set.v2i1.957

\begin{abstract}
Although fur artifacts are not numerous, they are relatively common in northern China's museums. This article focuses on the conservation of a man's court robe from the Palace Museum, lined with golden yellow silk kesi and decorated with golden dragons in colorful clouds and fur trim. It illustrates the treatment process for fur garments, including fur type identification, original sewing methodology documentation, replacement fur piece preparation and degraded fur repair. The fur class cultural relics appraisal method makes we completed a research project ${ }^{1}$. It based on fur fiber micro detection in the field of zoology $y^{2}$ and fiber detection of textile field ${ }^{3}$. Fur repair methods described in the article isfusion of the traditional fur production and restoration. This article would provide a detailed reference for future similar fur artifact repair work.
\end{abstract}

Keywords: fur, court robe, repair, fur type identification, kesi

\section{Foreword}

Fur artifacts are common in northern China's museums. However, their number is not numerous, especially for the case of fur garment. In the collections of the Palace Museums in Beijing, there are about thousands of fur garments preserved from the Qing Dynasty. These fur garments include fur robe, fur gown, fur waistcoat, mantle and dusihi etc. Some of these garments contain whole fur liner, and some of them contain fur edge (for example cuff, collar, top and bottom hem). The varieties of the fur include pelt from sable, fox, mouse, otter, sheep, lynx, deer, golden monkey and more than ten kinds of other animals.

These fur garments have been kept carefully in lead coated wooden boxes. The conservation condition is good, and almost all of them are perfect in appearance, except for vary few of them, which have been damaged. The causes of the damage mainly fall into two cases. The first case is caused by time, in which places had been damaged by insects become completely or spotted hairless. The second case is that the fur dyed black by fumigation has been degraded by the constituent of the dye.

The repaired "man's court robe lined with golden yellow silk kesi and decorated with golden dragons in colorful clouds and fur trim" is classified as a third-class cultural relic in the Palace Museums. It is a child court robe with total length of the two sleeves $133 \mathrm{~cm}$, robe length $91 \mathrm{~cm}$, bottom hem $110 \mathrm{~cm}$, and cuff $20 \mathrm{~cm}$. This court robe is lined with golden yellow silk kesi and decorated with golden dragons in colorful clouds outside, and lined with moon white silk threads inside. In its borders sable (Martes zibellina) was sewn. It was probably worn by child Emperor Tongzhi (1856-1875) or Emperor Guangxu (1871-1908) when they were young.

\section{Outer Fur Edge Repair}

\subsection{Conservation Plan}

The first part of the conservation is to access the conditions of the damaged fur edges. The type of the fur was inspected and identified before the fur edges were detached. Meanwhile, the original sewing methodology was examined and documented. Following that, the repair materials were chosen, processed and used to repair the fur edges by sewing. The second part is to consolidate the damaged cuffs. The last part is to fix the split threads in the ground silk (kesi ground fabric) as well as the stitching threads between liner and cuffs of the court robe.

The treatment of the court robe follows these principles: to restore this artifact, materials from the original court robe should be used as far as possible, except for the insects-damaged hairless fur which should be replaced. The detaching of the court robe should be limited to the greatest extent. The detached places should be strictly documented and 
restored according to the original methodology.

\subsection{Condition Assessment}

All of the fur embroidered the edges of the court robe has been heavily damaged by insects. The front fur edge was almost hairless and the fur on the rear border had serious losses. The dyed sable cuffs had been degraded by the dye and were nearly hairless, with slight splitting. The silk kesi ground fabric had suffered from minor splitting and the stitching threads were broken. The appearance of the robe had been greatly affected by all of these damages, which made it unsuitable for display.

2.3 Photography: Record the Whole Artifact as Well as Close-ups for the Damaged Places (Figure 1, 2).

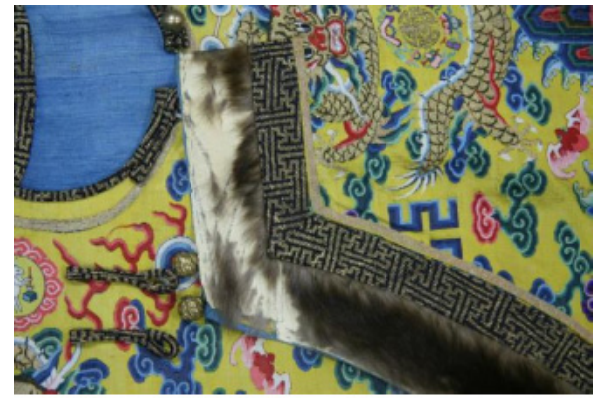

Figure1. The upper front fur edge of the court robe before repair

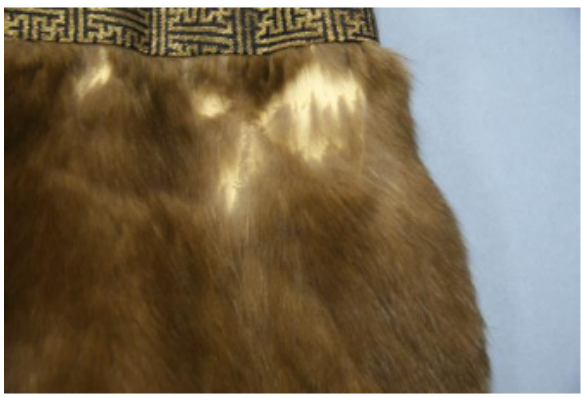

Figure 1. The bottom front hem of the court robe before repair (damaged aeras)

2.4 Documentations of the Damaged Parts: Documentations for the Front and Back Edges of the Court Robe, as Well as the Damaged Fur Edges (Figure 3, 4).

Legend: incomplete, breaking through, stitching threads broken, spilt

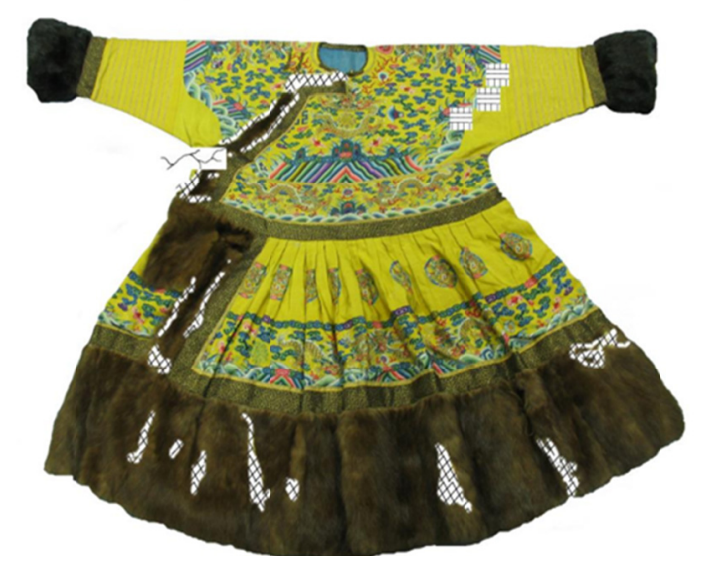

Figure 3. The front edge of the court robe

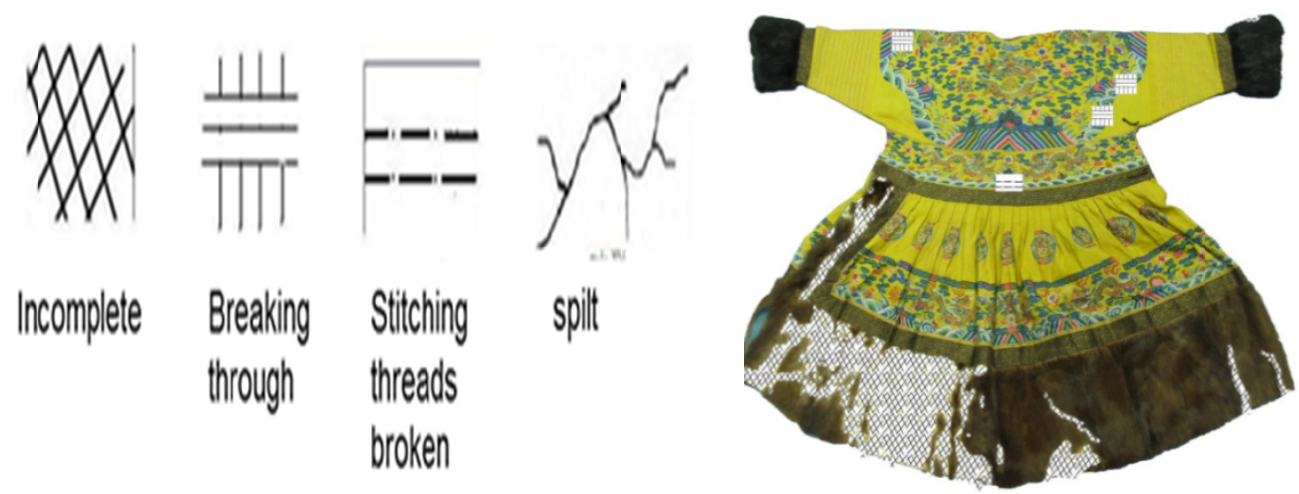

Figure 4 . The back of the court robe 


\subsection{Record: Record in Detail the Process of Detaching Fur Edges}

2.5.1 The Length of the Upper Front Fur Edge Was $10 \mathrm{~cm}$, the Width Was $5 \mathrm{~cm}$. One Place was Hairless. There Were Five More Places Slightly Lacked Hair (Figure 5).

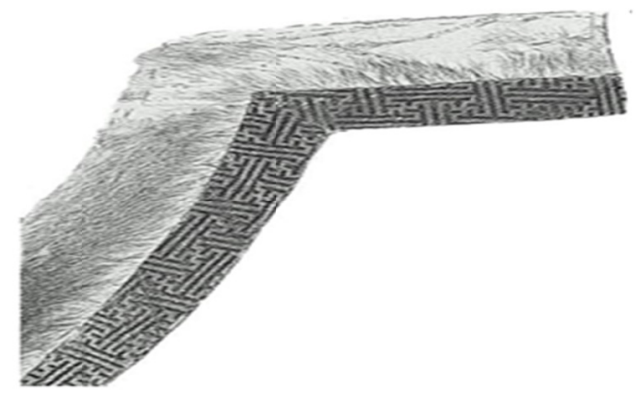

Figure 2.The upper front edge of the court robe

2.5.2 Bottom Hem of the Front Fur Edge: Length $111 \mathrm{~cm}$, Width 19-21 cm, Ten Hairless Places; (Figure 6)

\begin{tabular}{lllllllllll}
\hline$\#$ & 1 & 2 & 3 & 4 & 5 & 6 & 7 & 8 & 9 & 10 \\
\hline Length $(\mathrm{cm})$ & 3 & 5 & 2 & 2 & 1 & 4 & 1 & 2 & 2 & 1 \\
Width $(\mathrm{cm})$ & 3 & 4 & 2 & 1 & 3 & 1 & 1 & 1 & 11 & 2 \\
\hline
\end{tabular}

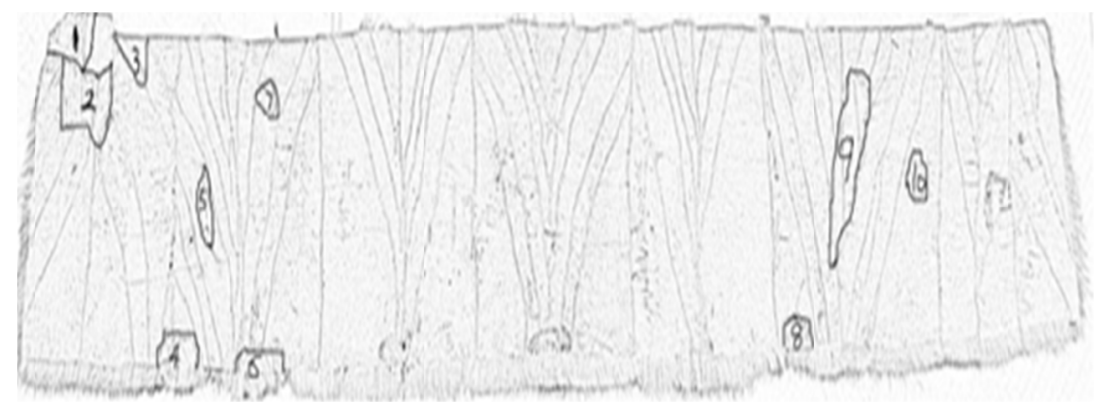

Figure 6. Bottom hem of the front fur edge

2.5.3 Bottom hem of the back fur edge: length $169 \mathrm{~cm}$, width $19-21 \mathrm{~cm} .86 \mathrm{~cm}$ of it which had little hair left had been removed from the fur edge. Some of the fur removed has been made into replace materials. The other $83 \mathrm{~cm}$ had six hairless places. (Figure 7)

\begin{tabular}{lllllll}
\hline$\#$ & 1 & 2 & 3 & 4 & 5 & 6 \\
\hline Length $(\mathrm{cm})$ & 2 & 8 & 4 & 2 & 1.5 & 14 \\
Width $(\mathrm{cm})$ & 3 & 7 & 5 & 6 & 2 & 3 \\
\hline
\end{tabular}

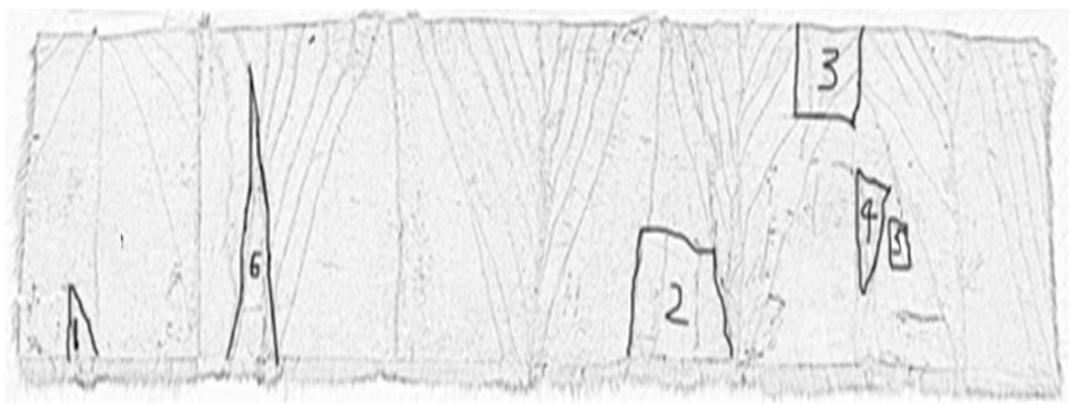

Figure 7. Bottom hem of the back fur edge (damaged part)

\section{Material Analysis ${ }^{1}$}

The common method used in fur type identification is the traditional sensory inspection method, in which analysts classify the fur type based on fur color, appearance, guard and ground hair texture as well as their experience. The fur type of this court robe was sable according to the archive. By our appearance inspection the characters of the fur was recorded. The guard hair of the fur was tawny while the ground hair was light grey. The length of the guard hair was 
about $2.5 \mathrm{~cm}$ while the length of the ground hair was $1-1.5 \mathrm{~cm}$. Moreover, the ground hair was scarce and the guard hair was flexible. These features match those of the sable. Except for referring to archive and appearance inspection, the fur type has also been confirmed with one of our other ongoing works. The fur type was identified by studying the microscopic structure of the fur, which is to exam the cross and longitudinal sections of the hair. Hair samples were taken from bottom hem of the fur edge, inside and outside of the sable cuffs respectively. Histology samples were made for cross section, longitudinal section and squama distribution. (Figure 8-12) The micro structure of the hair has the following features: the guard hair was composed of cortex layer and medulla layer; the squamae arranged densely and organized; some of the squamae were zigzag and clung closely to the hair shaft; the medulla layer was formed by bead shaped substructures arranged in one or multiple layers; the cross section of the medulla layer was elliptical; the uplift angle of the ground hair was huge, thus was distinct in characteristic; the cross section of the ground hair was almost round. All of the above characteristics agreed with the characteristics of the cross and longitudinal section of sable hair, as well as the characteristics of the distribution and ratio of sable hair squamae of different shapes. As a result, by integrating the appearance inspection and the micro structure study, we have drawn the conclusion that the fur edges and the fur inside of the cuffs were sable, while the outside of the cuffs were sable that has been dyed black.

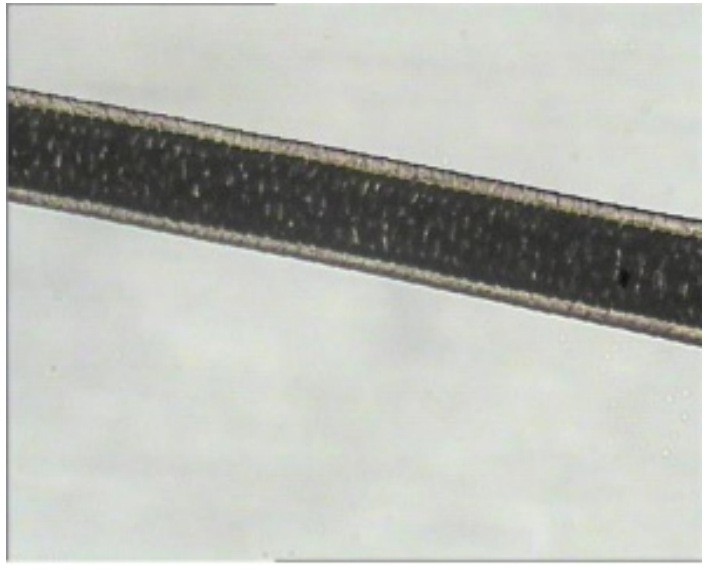

Figure 8. Bottom hem fur edge longitudinal section (1000X)

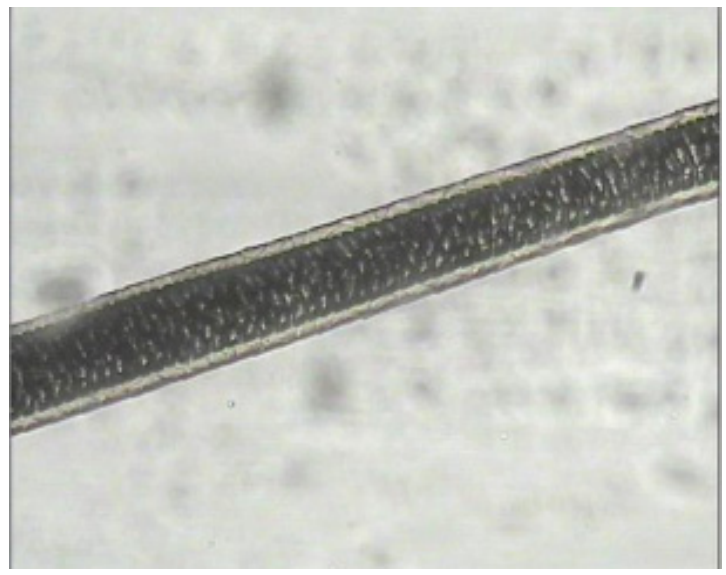

Figure 10. Inside of the cuffs fur edge $(600 \mathrm{X})$

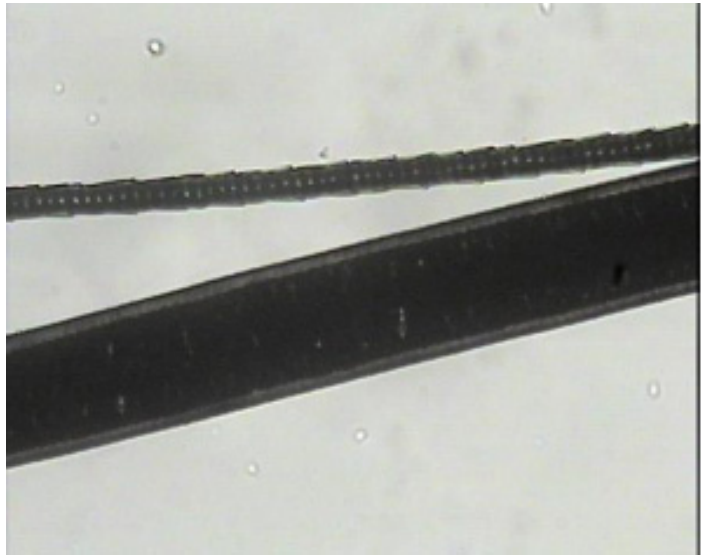

Figure 9. Outside of the cuffs fur edge $(600 \mathrm{X})$

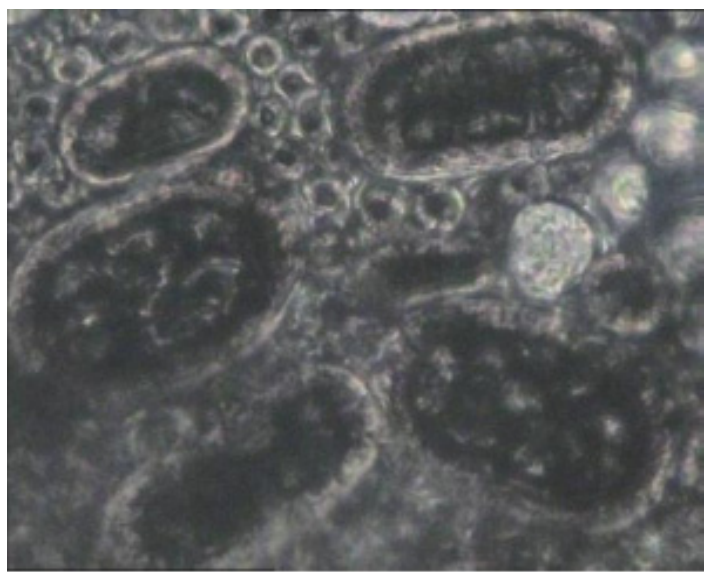

Figure 11. Bottom hem fur edge cross section $(1000 \mathrm{X})$ 


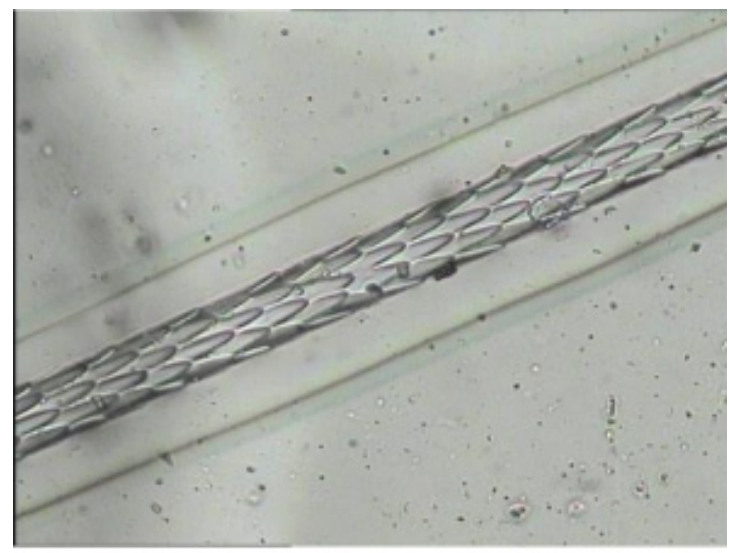

Figure 12. Front edge fur guard hair squama

\section{Detach Fur Edges}

All of the front and back fur edges (except cuffs) needed repair were detached. The original sewing methodology was recorded in detail.

4.1 Front Edges, Back Edges and Bottom Hem: the Sewing Methods of the Front and Back Edges Were the Same Yellow stitching threads were needled vertically through the back fur edges and the liner of the robe. There were 16 stitches along the bottom hem of the front edge and 24 along the bottom hem of the back edge. The sewing method was flat stitches. The distance between the yellow thread stitches was about $10-15 \mathrm{~cm}$ one stitch and was not universal. Horizontally, there were two yellow thread stitches needled, with about $6-7 \mathrm{~cm}$ separation. The sewing method was also flat stitches. The fur edges and the liner of the robe were combined together with the vertical and horizontal stitches described above. After detachment, the fur edges were dusted with a lint roller.

4.2 Sewing Methodology: The Sewing Methodology for the Fur Was Known as Ba Long

The fur was cut using a method called chuan dao, in which the fur was cut along the diagonal into strips of 4-5 $\mathrm{cm}$ in width. These tiny strips were then sewn into larger pieces. The assembled pieces of fur were large in size and thus suitable for making a garment. Ba long is a standard method in traditional fur garment making techniques. The edges and cuffs of the court robe were made by fur pieces assembled together following this method. As a result, though the sable fur pieces were not uniform in color, the hue of the whole fur edges was harmonious.

\section{Making Replacement Material}

The original fur on the court robe was wild sable, but now this species is nearly extinct and is also a nationally protected wild animal which it is forbidden to trade. Thus, it is impossible to use wild sable for replacement fur pieces. Through screening and comparison of the available fur types, the domestic brown mink was selected, as the color and texture of the guard hair of mink greatly resembles sable. Nevertheless, the brown mink is denser than sable, and has more ground hair and thicker, harder guard hair. Also, the brown mink was sleeker in hue thus was slightly different from the original sable. In result, apart from the regular treatment, it was necessary to pretreat the mink to imitate an aged look before using it for repairs. From the characteristics of the cross section of the brown mink hair (Figure 13, 14) it can be seen that, compared to the sable, the brown mink hair has thicker cortex layer but smaller medulla layer than the sable hair. These characteristics could explain the reason that the brown mink hair is coarser than the sable hair in touch.

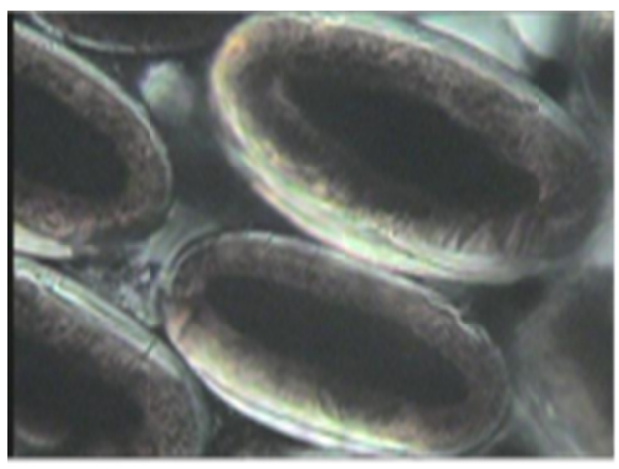

Figure 13. Cross section of the brown mink hair (1000X) (1000X)

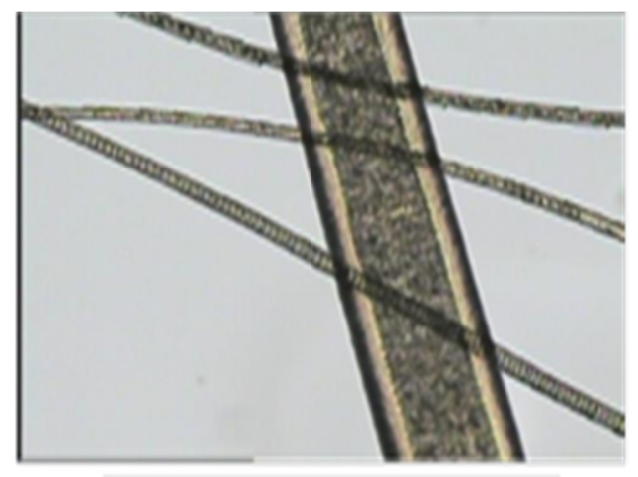

Figure 14. longitudinal section of the brown mink hair 
Choose Fur: Chose the Pelt Whose Hair Length, Hue and Color Were Close to the Original Fur Edges. The Chosen Pelt Should Be Soft, Flexible and Uniform.

Cut Fur (Figure 15): The Chosen Pelt Was Cut Vertically By Sharp Knife Along The Abdomen And Was Unfolded Into A Flat Piece Of Fur. The Cuts Should Be Made Steadily. Cares Should Be Taken At The Places Where The Cutting Started And Ended.

Process fur (Figure 16): Damages and flaws on the hairy side of the fur were checked and repaired if necessary. The parts with flaws was cut off, after which the rest of the fur was sewn together again. The sewing should be done with uniform, close stitches.

Flatten fur (Figure 17): The cut fur piece was flattened by a crescent moon shaped knife. The hairy side of the fur was laid on a surface. The crescent moon shaped knife was pressed against the other side of the fur while moving back and forward to stretch it.

Shape fur (Figure 18): Next step the fur was dampening with wet brushes. After fully dampening with water for a while, it was laid flat and dried in the shade, to make the processed fur stretch more complete and appear naturer. The fur was combed smoothly and tidily with coarse brushes after drying.

Imitate aged look: The fur pieces were tailored after having been processed following the above steps. (Figure 19) The escessive corners were cut out. The left fur pieces were trimed and treated to imitate aged look. After that, the fur pieces were ready to be used as replacement materials.

The new replacement fur pieces were densier and richer in ground hair compared to the original pieces. Most of the ground hair was removed with hairbrush to make the texture of the new pieces resemble the original one. (Figure 20)

Dye: A pale yellowish colored dye solution containing dye-fixing agent was made after trail and error. Coloring was performed by repeatedly appling the dye solution to the replacement fur pieces with hairbrush, meanwhile comparing them with the original fur pieces until their color mathces (Figure 21).

\section{Cut original Fur Edges}

The patterns of the hairless places of the original fur pieces were traced down on paper. Then, the hairless parts were cut out guided by the traced patterns. (Figure 22) All of the hairless places were cut out following this method. 


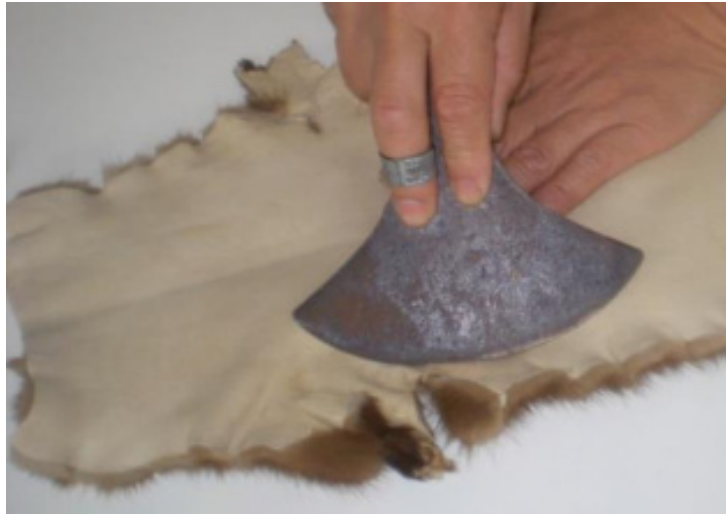

Figure 17. Flattening fur

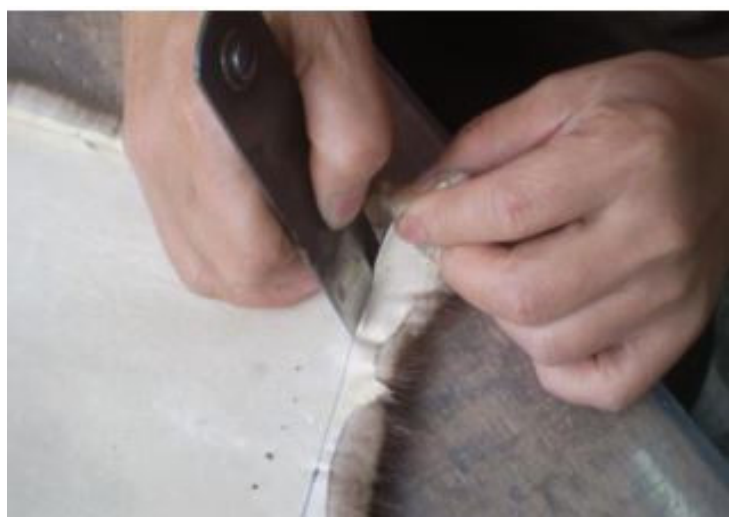

Figure 19. Making replacement materials

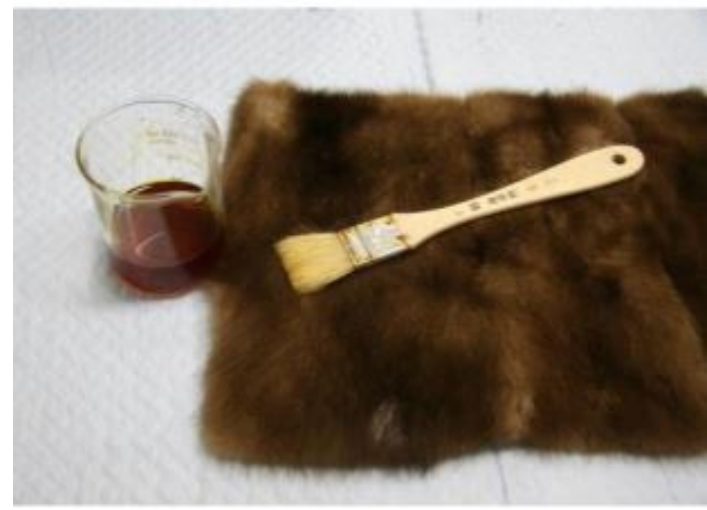

Figure 21. Dyeing fur

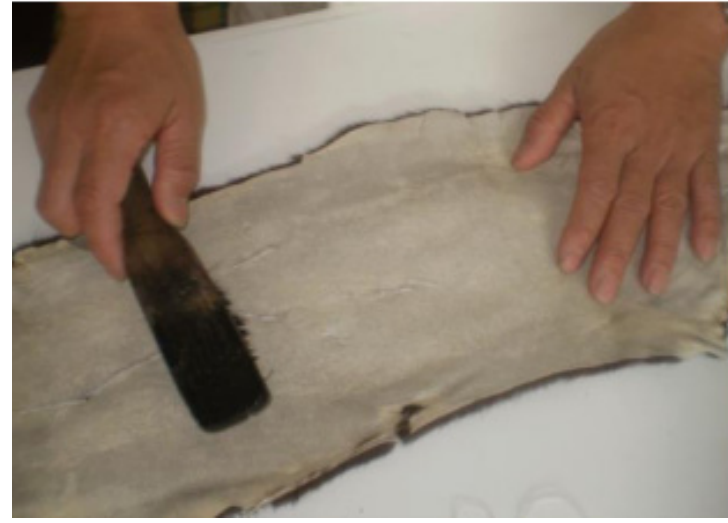

Figure 18. Dampening fur for shaping

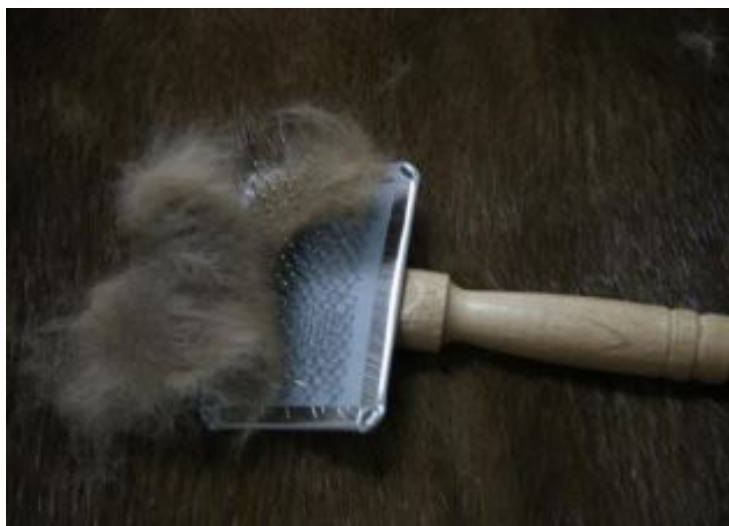

Figure 20. Removing excessive ground hair

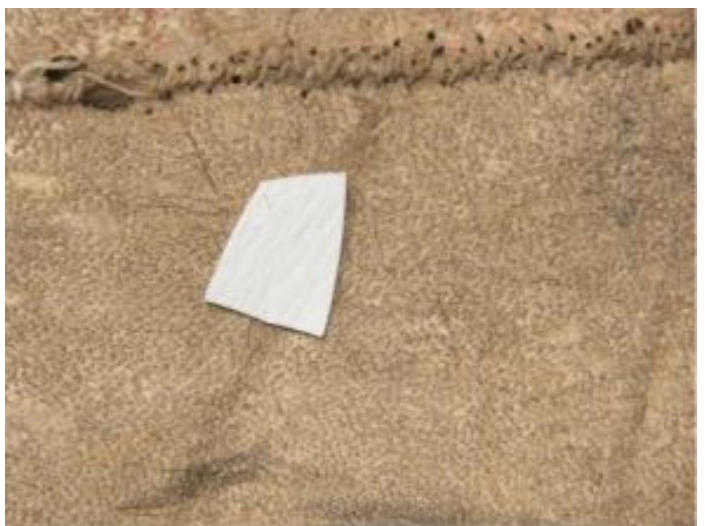

Figure 22. Cutting off hairless part

\section{Choose Infilling Materials}

The half left $86 \mathrm{~cm}$ of almost hairless fur edges of the bottom hem in the back was detached. The part with hair would be used as infilling materials. One other part at the rim of the left side of the bottom hem was also cut off for infiling materials, as it was heavily damged by insects. The infilling materials were trimed under the guidance of the paper mask. (Figure 23) The direction of the hair, and the area and color of the fur pieces were taken into consideration. All of the infilling materials for this court robe was made from the orignial pieces, so that the hue of the court robe was harmonious and the fur edges looked uniform.

\section{Sew Fur}

First the infilling material was fixed with pins at the hole in the cut original fur edges. (Figure 24)Then, the infilling materials were sewn to the original fur edges with back slip stitches. The distance between the stitches when sewing 
should not be the same, in case that the aged fur edges break along the stitches. Cotton threads were used, which was the same as the original threads. (Figure 25) The inside of the bottom fur edge of the front of the court robe was intact, thus was relocated into the back of the court robe. The $86 \mathrm{~cm}$ new fur piece that had been treated was connected into the back of the original fur edge, so that the new fur piece would be hided at the inside of the fur edges. The infilling materials for the bottom hem at the front and the back of the court robe were all made from the original fur pieces. When displayed, the repaired court robe would have excellent appearance, as all of the parts could be seen except the left side at back would be the original sable.

\section{Shape the Fur}

The repaired fur edge was soaked with water (Figure 26), rolled up (Figure 27), and left until it was completely wet, so that the stiff fur was able to regain its flexibility. It was then opened and dried in the shade. The fur was combed smoothly and tidily with coarse brushes after drying.

The hairless $10 \mathrm{~cm}$ by $5 \mathrm{~cm}$ part at the top of the front edges of the court robe was replaced with replacement materials made from the fur pieces from the left back fur edges. The left back fur edges were replaced by processed aged looked new fur.

\section{Re-sew the Stitches}

Silk threads with the same diameter of the original threads were chosen. The threads were dyed and sewn into places with stitch style same as the original stitches. (Figure 28)

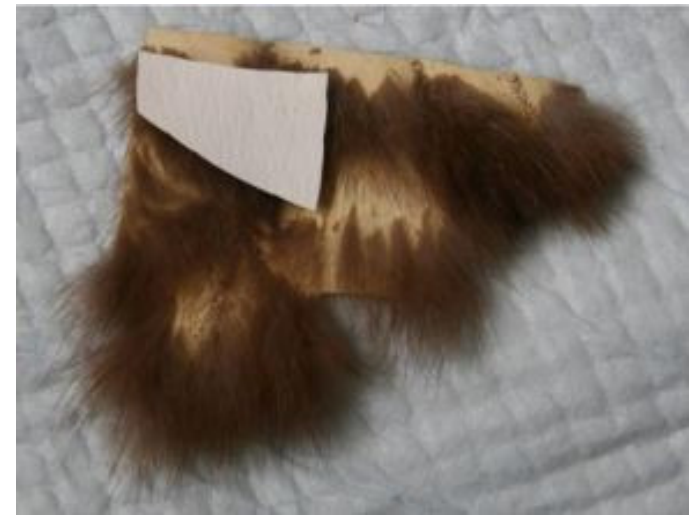

Figure 23. Choosing infilling materials

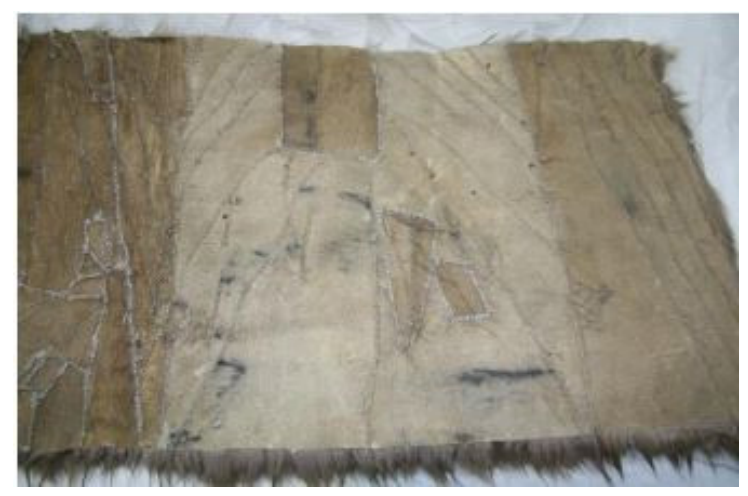

Figure 25. After sewn

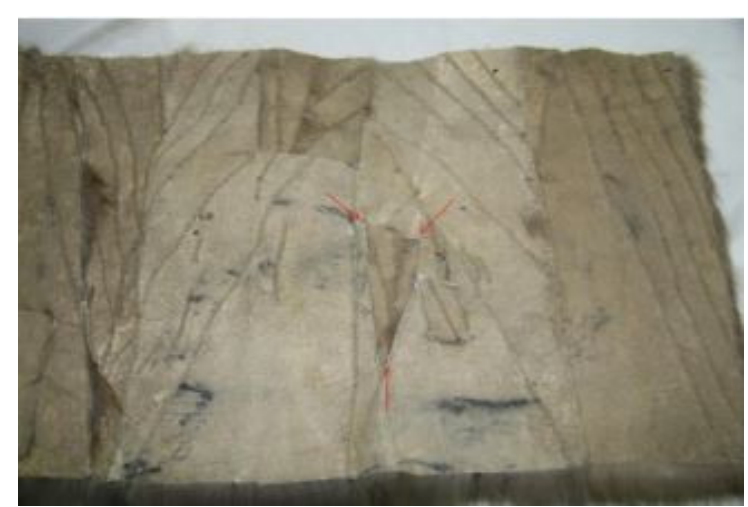

Figure 24. Fixing the infilling materials

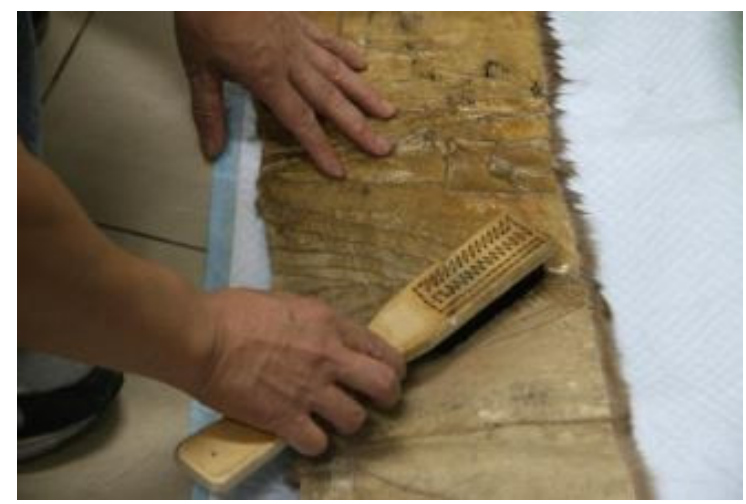

Figure 26. Dampening fur 


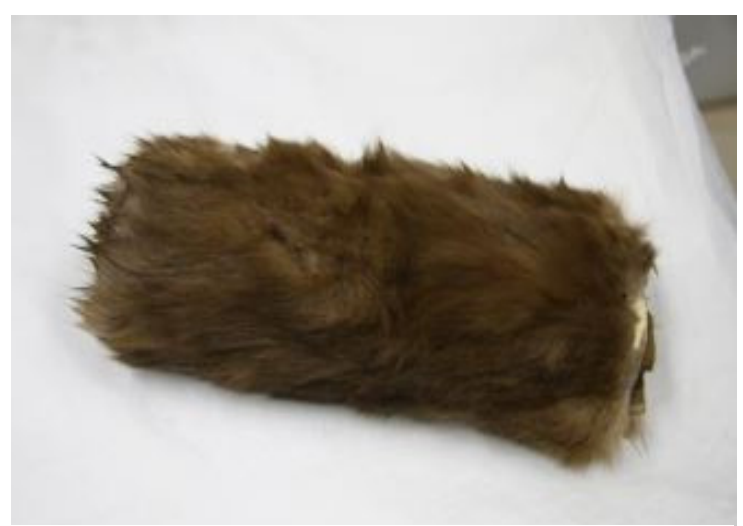

Figure 27. Soaking fur

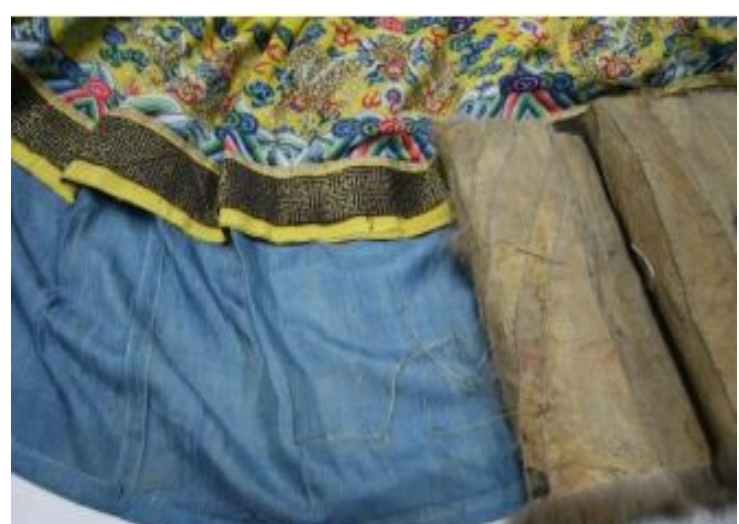

Figure 28. Re-sewing fur edges

\section{Cuffs Repair}

Conservation plan: The fur edges of the cuffs were made from black dyed sable. Due to the corrosion of the dye, the hair of the fur was loosened. Though detaching the cuffs would make consolidation easier, it could damage the information of the original technology by which the cuffs were sewn. As a result, the consolidation was carried out with the cuffs remained on the court robe.

There were three damaged places having been repaired of the two cuffs (Figure 29):

Place number 1 had a small fur piece detached, and it was sewn back.

Place number 2 had lost $1 \mathrm{~cm}$ by $2 \mathrm{~cm}$ of fur. Infilling materials made from new fur was used to fill it.

Place number 3 had broken stitches. Repair has been made by sewing.

Repair method: A big piece of hard cloth was placed at the bottom of the fur cuffs from the opening of the hole. This piece of cloth was sewn together with the detaching fur piece with running stitch to consolidate it. Because the fur has been weakened over time, the threads used should be neither too thick nor too thin, the spaces between stitches should be adequate, and the stitches should be placed at relatively strong places.

\section{Ground Silk Kesi (Ground Fabric) Repair, Sew Stitches}

Ground sile kesi (ground fabric) repair: There were six spilt threads in between the colour pattern on the front and back of the robe and the yellow silk fabric.The area that needed repair is very small. We have used needle and dyed thin silk threads to restore it following the original stitching style for kesi (Figure 32, 33).

Sew stitches: The broken threads on the back waist areas were re-stitched using the original stitching holes with dyed silk threads of the same thickness. 


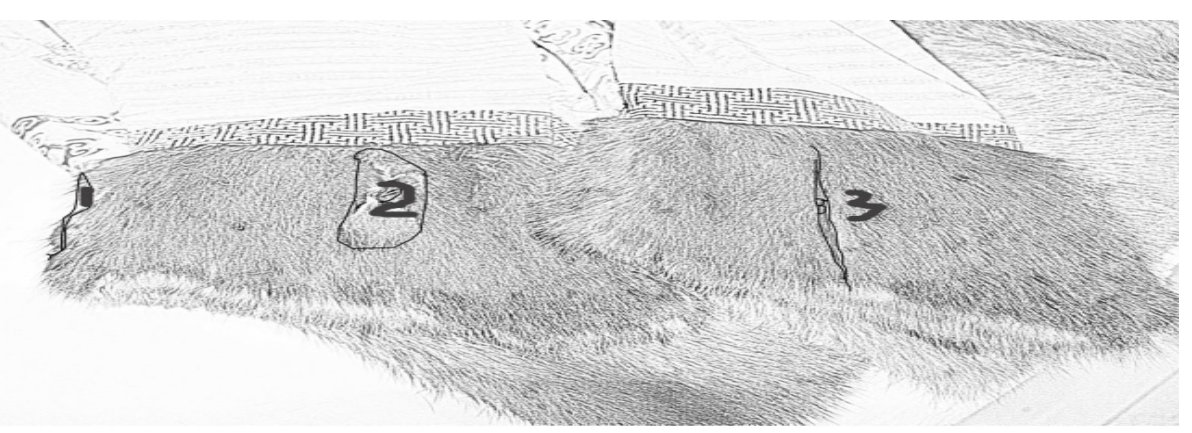

Figure 29. Schematic diagram for damaged cuffs

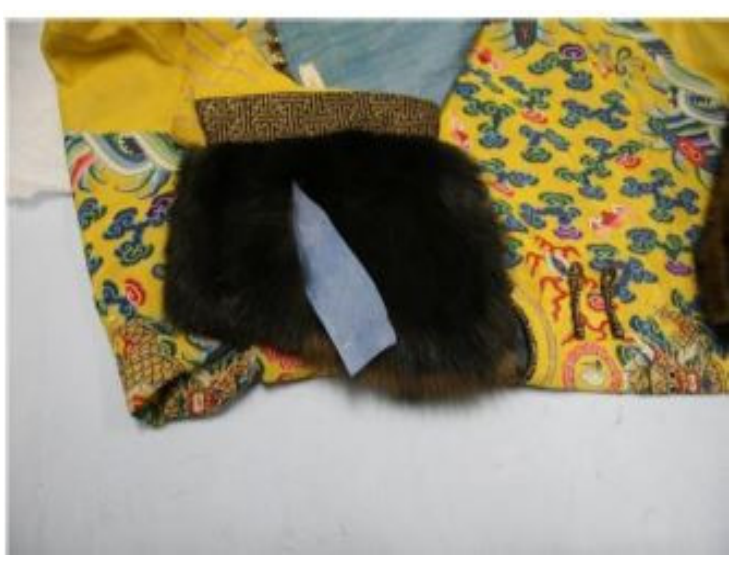

Figure 30. Lay backing cloth

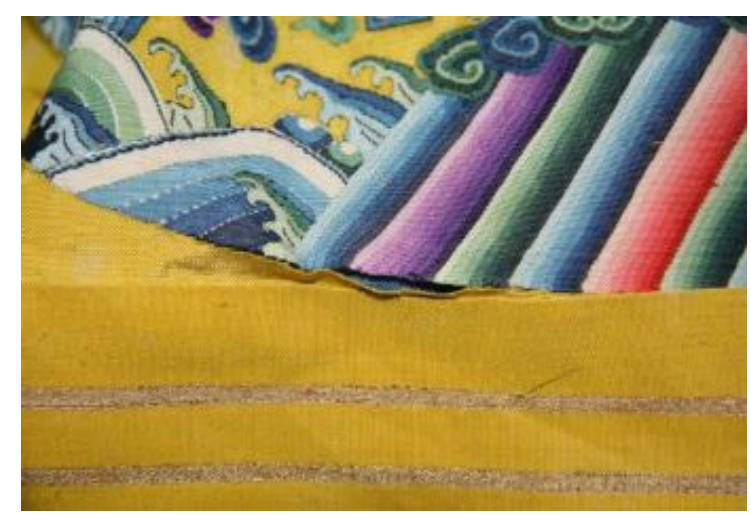

Figure 32. Split kesi

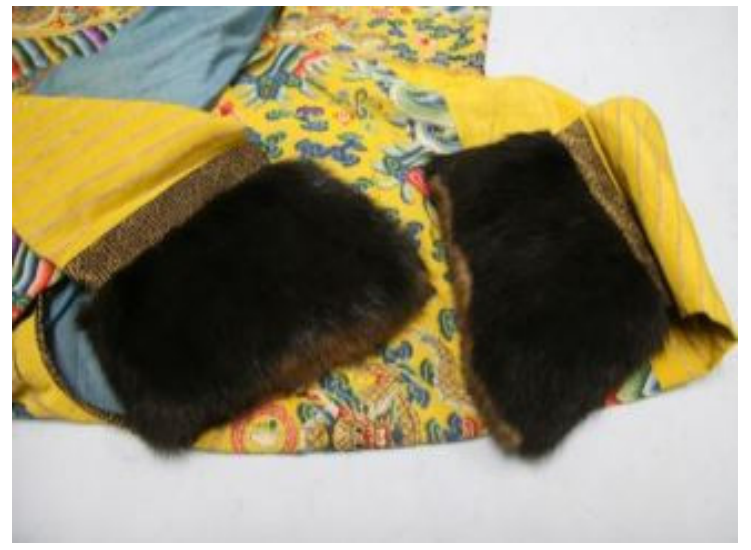

Figure 31. Repaired cuffs

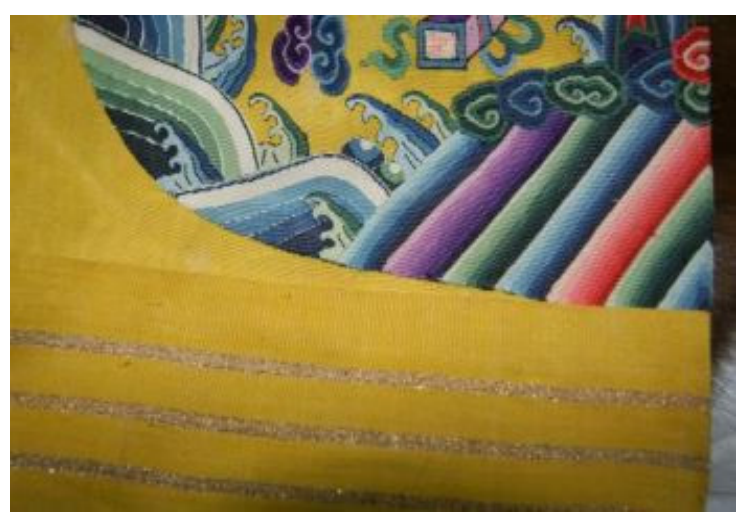

Figure 33. Repaired kesi 


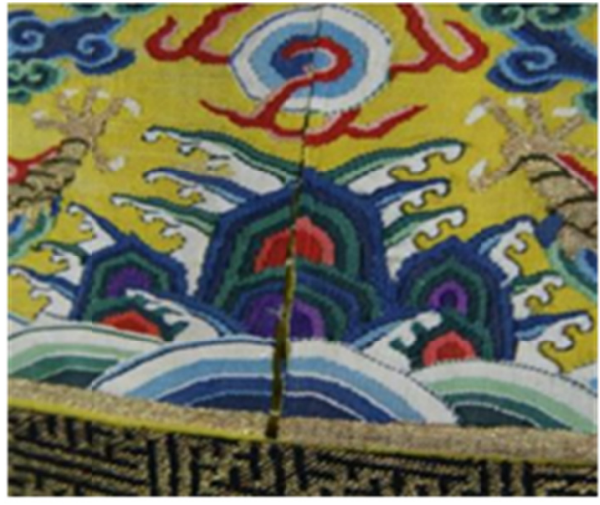

Figure 34. Split threads

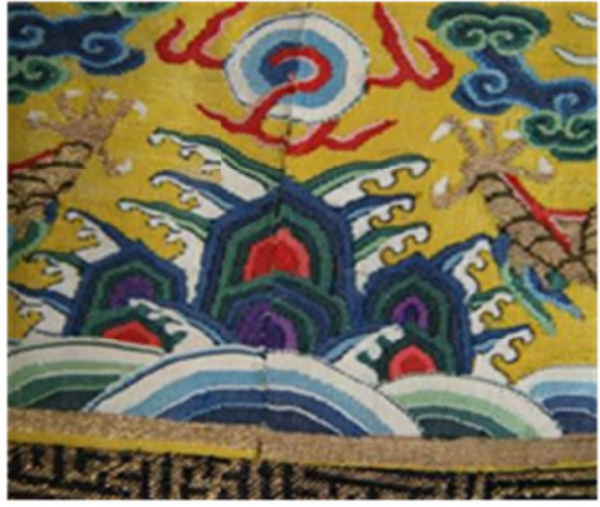

Figure 35. Sewn stitches

\section{Summary}

This article introduces the repair procedures for fur garments. The main steps are: damage condition investigation, materials identification, stitch style analysis and infilling materials preparation and so on. The original stitch style and sewing technology has been analyzed and documented, via detaching the damaged fur edges from the court robe. To identify the fur type, the micro structure of the fur hair was examed by micro section, which is one of our ongoing projects. The replacement materials made from new fur have been pretreated to imitate an aged look apart from treated with other regular procedures, so that the repaired court robe looks harmonious. The sewing techniques for new fur were borrowed to combine with the textile conservation method, and the original fur was used as far as possible. As a result, an improved and innovative idea has been developed for repairing old and decayed fur by employing traditional fur sewing techniques. A preliminary set of handicraft techniques for conserving decayed and insect-damaged fur artifacts has thus been formulated. To our best knowledge, this is the first reported work for fur artifact conservation. This article would provide a detailed reference for future similar fur artifact repair work.

\section{ReferenceS}

Gan, S. (1992). Secience and Technology Press, 53-62.

Gao, Y. Q .Color Histological Atlas of the Animal Hair [M]

Hei, L. J. (2002). Northeast Forestry University press, 27-52.

The Chinese chemical society committee of applied chemistry and Chemical scientific committee for the protection of cultural relics and archeology. The research results of the protection of cultural relics roundup . Anhui science and technology publishing house .2015.14-18.

Wang, Y. L. Institute of identification method of fur[C]

Zhang, W., Jing, S. Y., Tu, Y. C. Fur secience[M]

\section{(c) EY}

This work is licensed under a Creative Commons Attribution 3.0 License. 